\title{
EDITORIAL
}

\section{Wilderness First Aid: Managing Remote Area Medical Issues}

An article in this edition of Wilderness and Environmental Medicine by Welch, Clement, and Berman discusses several aspects of the issue of "wilderness first aid" courses that are intended to increase awareness in lay audiences concerning best practices for managing remote area medical issues. Typical courses include Wilderness First Aid (WFA), Wilderness First Responder (WFR), and Wilderness Emergency Medical Technician (WEMT or EMT-W) and a number of hybrid courses that teach a combination of delayed transport medical care and other skills, such as leadership, survival, and/ or situation command and control.

The first issue evaluated is part of the title (ie, "Is There an "Industry Standard""). The authors respond with a strong "no" based upon a number of criteria. One criterion cited in the article is that "providers" of this instruction have repeatedly claimed that the material they are teaching is proprietary. The Wilderness Medical Society (WMS) attempted to standardize WFA during 1999 and, indeed, met rejection of this effort by the leadership of many of the major providers. The issue of proprietary property was their excuse for noncooperation with this effort.

This article also raises another question having to do with the validity of content. With no national standard the term "wilderness first aid" has taken on a life of its own. Literally anyone can teach anything he wishes and, as long as the program lasts 12 to 16 hours for a WFA or 60 to 80 hours for a WFR, it appears to satisfy the requirements of the consumer. Or does it? This article demonstrates a lack of outcomes-based content, a doubt that the depth of coverage taught by most courses can be adequately retained by the lay student, and the high cost of obtaining such helter-skelter training.

During the 25th Annual Meeting of the Wilderness Medical Society held in Snowmass, CO, in July 2008, an elegant presentation was given by Arthur A. Islas, MD, MPH, FAWM, which demonstrated the use of the
Haddon's Matrix, a validated method for risk conceptualization and development of injury prevention methods, which Islas applied to wilderness-related injuries documented through an extensive review of the literature. As Dr Islas demonstrated, the methodology exists to develop a curriculum for wilderness first aid training that is literature based and not simply expert opinion based.

One topic this article did not broach was the issue of legality. Even if outcomes-based, national standard WFA, WFR, and WEMT courses could be devised and accepted by both providers and consumers of this education, such courses could still face severe legal criticism. Recently the Risk Management Committee of the Student Conservation Association revised its epinephrine protocols to comply with the majority of state laws concerning the use of epinephrine and thereby veered from commonly taught practices contained in most wilderness first aid courses. This is possibly a trend that might have to continue as boards of various organizations come to realize that some protocols advocated by this unregulated and nonstandard industry of wilderness first aid instruction can run afoul of state medical practice licensing laws.

It is time for the wilderness medical community to bring together experts in education, recreational and medical law, and wilderness medicine epidemiology to design a standard wilderness first aid curriculum-one that will provide the end users of this education with a cost-effective, retainable, and appropriate wilderness prehospital-care training experience. The WMS has the expertise, the energy, and the credibility to be the focus of this effort. Perhaps it is time to revisit the WMS project that was attempted in 1999.

William W. Forgey, MD

Past President, WMS Merrilville, Indiana 